\title{
Tailoring non-viral delivery vehicles for transporting genome-editing tools
}

\author{
Wujin Sun ${ }^{1,2}$ and Zhen $\mathrm{Gu}^{1,2,3^{*}}$
}

\begin{abstract}
The CRISPR-Cas system, especially the type II CRISPR-Cas9 system from Streptococcuspyogene, has rapidly emerged as a popular genome editing tool. The development of Cas9 derivatives further expanded the toolbox of CRISPRCas9 based genome editing kit. However, therapeutic translation of the CRISPR-Cas9 system in vivo is severely impeded by the absence of an appropriate delivery carrier. The complexity and high molecular weight of the CRISPR-Cas9 system, together with the physiological barriers for nucleus targeted cargo transportation have made it a huge challenge for in vivo therapeutic CRISPR-Cas9 delivery. Currently, the main stream carriers for systemic delivery of CRISPR-Cas9 are viral based, such as adeno-associated virus. However, the safety concerns surrounding viral vectors call for the development of non-viral nanocarriers. In this review, we survey the recent advances in the development of non-viral delivery systems for CRISPR-Cas9. Challenges and future directions in this field are also discussed.
\end{abstract}

Keywords: CRISPR-Cas9, drug delivery, gene therapy, nanomedicine, genome editing

\section{INTRODUCTION}

The promise of treating disease from the genetic roots made gene therapy an actively pursued modality by both scientists and the general public [1-4]. The therapeutic effect is achieved by applying genetic tools to precisely manipulate genetic materials within targeted cells, including disruption, insertion, deletion, mutation and replacement [5]. Ongoing clinical trials of gene therapy shed light on treating serious genetic diseases associated with different physiological systems, such as the immune, nervous or blood circulation systems [6]. The type II CRISPR-Cas (clustered regularly interspaced short palindromic repeats (CRISPR)-CRISPR associated protein) system has rapidly emerged as a facile genetic engineering tool for targeted interrogation of almost any gene in any organism $[7,8]$. Early investigations coined the popular CRISPR-Cas9 system from Streptococcus pyogenes as a site-specific endonuclease that could bind with a single guiding RNA (sgRNA) to recognize specific DNA sequences for cleavage [9]. Cleavage of the targeted DNA loci in the genome introduces double stranded DNA breaks, which could be repaired by the cells through different pathways. Typically, DNA repair of the cleavage introduces insertions or deletions (indels) through the error-prone non-homologous end joining pathway (NHEJ), a mechanism usually adopted for targeted gene disruptions [10]. In the presence of a pre-designed donor DNA, precise genome editing could occur incorporating sequence from the donor DNA into the genome by homologous recombination [11]. The site-specific nuclease activity makes CRISPR-Cas9 a powerful tool in achieving permanent changes to the genomes. Further development of Cas9 by disabling its enzymatic activities generates a "dead" version of Cas9, termed as dCas9, that could be fused with different functional protein domains to achieve transcriptional control, epigenetic regulation or imaging [12].

Delivered by traditional gene transfection methods, such as mechanical deformation of cell membrane [13], electroporation [14], lipofection [15], microinjection [16] or viral based transfection [17], CRISPR-Cas9 system were widely adopted in ex vivo editing of germline cells from different species for the purpose of curing genetic disease in

\footnotetext{
${ }^{1}$ Joint Department of Biomedical Engineering, University of North Carolina at Chapel Hill and North Carolina State University, Raleigh, NC 27695, USA

${ }^{2}$ Division of Pharmacoengineering and Molecular Pharmaceutics and Center for Nanotechnology in Drug Delivery, Eshelman School of Pharmacy, University of North Carolina at Chapel Hill, Chapel Hill, NC 27599, USA

${ }^{3}$ Department of Medicine, University of North Carolina School of Medicine, Chapel Hill, NC 27599, USA

*Corresponding author (email: zgu@email.unc.edu)
} 
the off-springs of genetically diseased rats [18], reprogramming mice $[19,20]$ or monkey zygotes [21] to create animal models for scientific study, or even the controversial human germline cells for therapeutic studies [22,23]. However, these proof-of-principle studies may not be directly translated into therapeutic applications [24]. Recent therapeutic application of CRISPR is an indirect approach that focused on the ex vivo editing of immune cells for cancer immune therapy, like chimeric antigen receptors $\mathrm{T}$ (CAR-T) cells [25]. However, to realize the prospect of curing hereditary genetic disease in animals after birth, the development of a suitable delivery method is the number one challenge $[26,27]$.

\section{NONVIRAL CARRIER-MEDIATED CRISPR DELIVERY}

Many obstacles exist for delivering the CRISPR-Cas9 system: 1) Cas9 is a large protein $(\sim 160 \mathrm{kDa})$ with genetic sequence over 4000 base pairs (bp), making it an overwhelmingly huge load even for the well-established adenoassociated virus (AAV) based carriers [28]; 2) CRISPRCas9 is multicomponent system composed of Cas9 protein, sgRNA and an optional donor DNA, which makes it desirable to create carriers capable of delivering the three different types of biomolecules simultaneously; 3 ) CRISPR-Cas9 takes effect in the nuclei of cells, and to reach this destination, multiple physiological barriers need to be overcome, such as avoiding proteases and nucleases in blood circulation, extravasation into desired tissue, penetrating the plasma membrane of targeted cells, escaping the endolysosome entrapment after endocytosis, and infiltrating the nuclear membrane [29].

In contrast to the facile adaptation of almost all types of viral carriers to fit the CRISPR-Cas9 system [30], it has been a tough task to design efficient non-viral nanocarriers for systemic administration. In the first demonstration of CRISPR based therapeutic genome editing in adult animal, Anderson and coworkers [11] directly administered a plasmid encoding the Cas9 and sgRNA with a single-stranded DNA (ssDNA) donor to correct a single point mutation associated disease (hereditary tyrosinemia type I, HTI) in mouse liver by hydrodynamic injection via the tail vein. A $0.4 \%$ initial gene correction efficacy was achieved by this method. Recently, a core-shell structured polymeric nanocarrier has been reported to deliver the all-in-one plasmid for gene disruption in cancer therapy [31]. The fluorinated polymer core and hyaluronic acid shell based formulation induced $44 \%$ gene disruption in vitro and reduced targeted gene expression in vivo after intraperitoneal drug administration.

An alternative to the delivery of nucleic acid based CRISPR-Cas9 system is to deliver the CRISPR-Cas9 components as purified protein and RNA. Direct delivery of the ribonucleoprotein would save the time for cells to express them from the all-in-one plasmid, facilitating the onset of the gene editing process [32]. The absence of a plasmid DNA when delivering the ribonucleoprotein would obviate the concern of integrating plasmid into the genome of targeted cells [33]. It has been demonstrated that the Cas9/sgRNA ribonucleoprotein delivery strategy enabled better control of Cas9/sgRNA dosage and reduced the chance of off-target cleavage when compared with the plasmid delivery strategy [34]. Kim and coworkers [35] applied a cell penetrating peptide with 9 Arginine (9R) to deliver purified Cas9 and sgRNA (Fig. 1a). Cas9 was conjugated to $9 \mathrm{R}$ via a redox cleavable linker while sgRNA was complexed with 9R through electrostatic interaction. After co-administration of these two nanoparticles, $8.7 \%$ gene disruption was achieved in a HEK293T cell line. Besides the highly positively charged peptide, cationic lipids were also applied for Cas9/sgRNA ribonucleoprotein delivery $[32,36]$. The Cas9/sgRNA ribonucleoprotein has a net negative charge, making it easy to form stable nano-complex with the cationic lipids. A synthetic redox responsive lipid nanocarrier showed $70 \%$ gene disruption in a reporter cell line [36], and a commercially available lipid based carrier (Fig. 1b) has generated $20 \%$ gene modifications in an in vivo hair cell model after local injection [32].

Our group has recently developed a synthetic DNA nanoclew (NC) based carrier for delivering the CRISPR-Cas9 system in vitro and in vivo for gene disruption [37]. As shown in Fig. 1c, the DNA NC containing sequence complementary to the targeting region of sgRNA was prepared by a facile DNA synthesis technique named rolling circle amplification [38]. Purified Cas9 protein and sgRNA formed a ribonucleoprotein that could bind to the DNA NC via specific base-pairing between sgRNA and DNA NC. After loading Cas9/sgRNA into the DNA NC, a positively charged polymer polyethylenimine (PEI) was coated onto the nanoparticle for facilitated endosome escape. The final formulation containing the DNA NC core, Cas9/sgRNA cargo and a PEI coating formed uniformly sized nanoparticles with a mean hydrodynamic size of $56 \mathrm{~nm}$, which was an appropriate size for efficient drug delivery. A model bone osteosarcoma cell line expressing a destabilized version of EGFP (U2OS.EGFP) was chosen as reporter cell line [39]. Optimized DNA NC with partial complementarity to the sgRNA showed the highest Cas9/ 
a

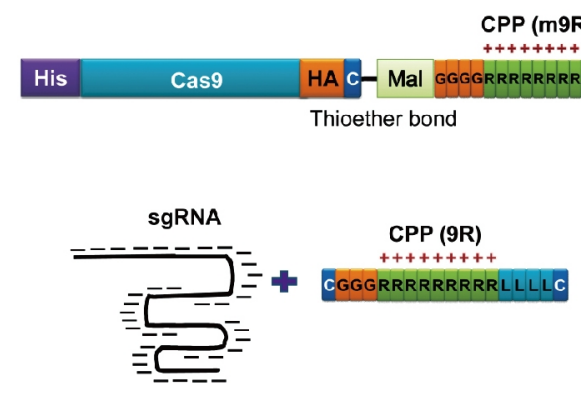

b

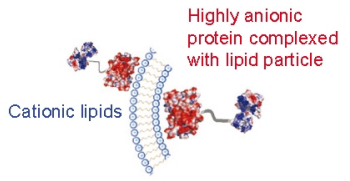

Treatment of

mammalian cells with cationic lipid:anionic protein complexes

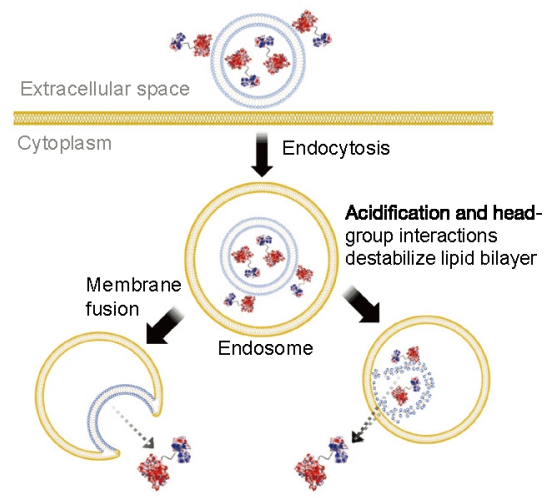

d

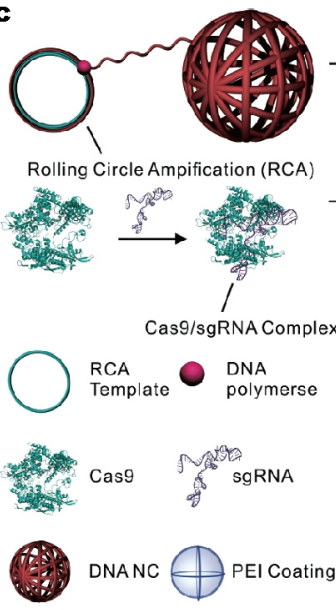

c

igure 1 Recently developed nonviral systems for CRISPR-Cas9 delivery. (a) CPP mediated delivery of Cas9 protein and sgRNA, adapted with permission from [35]. (b) Cationic lipid for delivering Cas9/sgRNA ribonucleoprotein complex, adapted with permission from [32], Copyright 2014, Nature Publishing Group. (c) A DNA nanoclew based carrier was tailored via nucleic acid complementation for delivering the Cas9/sgRNA ribonucleoprotein, adapted with permission from [37], Copyright 2015, Wiley-VCH Verlag GmbH \& Co. KGaA. (d) Lipid nanoparticle for delivery of Cas9 mRNA in combination with AAV delivered sgRNA and DNA donor, adapted with permission from [40], Copyright 2016, Nature Publishing Group.

sgRNA delivery efficacy, disrupting EGFP gene in $37 \%$ of the treated population in vitro. We also investigated the in vivo gene editing efficacy of Cas9/sgRNA delivered by the DNA NC based carrier. In a xenograft tumor model built with the U2OS.EGFP cell line, 25\% of the U2OS.EGFP cells in the locally treated region lost EGFP expression after 10 days of the treatment while cells in the untreated group all remained EGFP positive.

Besides using non-viral carriers only, combined non-viral and viral carriers were also demonstrated for enhanced genome editing efficacy. With the HTI as model disease, modified mRNA of Cas9 was encapsulated into a C12-200 based lipid nanoparticle, while the sgRNA and donor DNA was packaged into AAV (Fig. 1d). Single administration of the lipid complexed mRNA could lead to $77 \%$ gene disruption in a reporter cell line, while the combined viral and non-viral therapy induced $6 \%$ gene correction in the targeted hepatocytes after intravenously injection [40].

\section{CONCLUSIONS}

In summary, the task of delivering the huge and complex CRISPR system for therapeutic applications in vivo has remained challenging, and the race to develop an efficient and biocompatible non-viral nanocarrier has just begun. Currently reported non-viral nanocarriers share the common feature of adopting cationic materials. However, these cationic components could interfere with serum proteins if administered intravenously, which might compromise their therapeutic efficacy as well as biocompatibility. To develop a better nanocarrier, more biocompatible biomaterials that could efficiently package the multicomponent CRISPR into one nanocarrier and escape the endosome entrapment for efficient nuclear delivery are needed $[41,42]$.

Received 8 November 2016; accepted 13 December 2016; published online 28 December 2016

1 Philippidis A. Gene therapy briefs. Human Gene Therapy, 2014, 
25: 92-95

2 Walsh CE, Batt KM. Hemophilia clinical gene therapy: brief review. Translational Res, 2013, 161: 307-312

3 Simonato M, Bennett J, Boulis NM, et al. Progress in gene therapy for neurological disorders. Nat Rev Neurol, 2013, 9: 277-291

4 Evans $\mathrm{CH}$, Huard J. Gene therapy approaches to regenerating the musculoskeletal system. Nat Rev Rheumatol, 2015, 11: 234-242

5 Hotta A, Yamanaka S. From genomics to gene therapy: induced pluripotent stem cells meet genome editing. Annu Rev Genet, 2015, 49: 47-70

6 Naldini L. Gene therapy returns to centre stage. Nature, 2015, 526: 351-360

7 Doudna JA, Charpentier E. The new frontier of genome engineering with CRISPR-Cas9. Science, 2014, 346: 1258096-1258096

8 Heidenreich M, Zhang F. Applications of CRISPR-Cas systems in neuroscience. Nat Rev Neurosci, 2015, 17: 36-44

9 Jinek M, Chylinski K, Fonfara I, et al. A programmable dual-RNAguided DNA endonuclease in adaptive bacterial immunity. Science, 2012, 337: 816-821

10 Yang L, Güell M, Niu D, et al. Genome-wide inactivation of porcine endogenous retroviruses (PERVs). Science, 2015, 350: 1101-1104

11 Yin $\mathrm{H}$, Xue W, Chen S, et al. Genome editing with Cas9 in adult mice corrects a disease mutation and phenotype. Nat Biotechnol, 2014, 32: 551-553

12 Dominguez AA, Lim WA, Qi LS. Beyond editing: repurposing CRISPR-Cas9 for precision genome regulation and interrogation. Nat Rev Mol Cell Biol, 2015, 17: 5-15

13 Han X, Liu Z, Jo MC, et al. CRISPR-Cas9 delivery to hard-to-transfect cells via membrane deformation. Sci Adv, 2015, 1: e1500454-e1500454

14 Lin S, Staahl BT, Alla RK, et al. Enhanced homology-directed human genome engineering by controlled timing of CRISPR/Cas 9 delivery. eLife, 2014, 3: e04766

15 Polstein LR, Gersbach CA. A light-inducible CRISPR-Cas9 system for control of endogenous gene activation. Nat Chem Biol, 2015, 11: $198-200$

16 Sommer D, Peters A, Wirtz T, et al. Efficient genome engineering by targeted homologous recombination in mouse embryos using transcription activator-like effector nucleases. Nat Commun, 2014, 5: 3045

17 Tabebordbar M, Zhu K, Cheng JKW, et al. In vivo gene editing in dystrophic mouse muscle and muscle stem cells. Science, 2016, 351: $407-411$

18 Yoshimi K, Kaneko T, Voigt B, et al. Allele-specific genome editing and correction of disease-associated phenotypes in rats using the CRISPR-Cas platform. Nat Commun, 2014, 5: 4240

19 Platt RJ, Chen S, Zhou Y, et al. CRISPR-Cas9 knockin mice for genome editing and cancer modeling. Cell, 2014, 159: 440-455

20 Mizuno S, Dinh TTH, Kato K, et al. Simple generation of albino C57BL/6J mice with G291T mutation in the tyrosinase gene by the CRISPR/Cas9 system. Mamm Genome, 2014, 25: 327-334

21 Niu Y, Shen B, Cui Y, et al. Generation of gene-modified cynomolgus monkey via Cas9/RNA-mediated gene targeting in one-cell embryos. Cell, 2014, 156: 836-843

22 Liang P, Xu Y, Zhang X, et al. CRISPR/Cas9-mediated gene editing in human tripronuclear zygotes. Protein Cell, 2015, 6: 363-372

23 Bosley KS, Botchan M, Bredenoord AL, et al. CRISPR germline engineering-the community speaks. Nat Biotechnol, 2015, 33: $478-486$
24 Lundberg AS, Novak R. CRISPR-Cas gene editing to cure serious diseases: treat the patient, not the germ line. Am J Bioethics, 2015, 15: $38-40$

25 Ren J, Liu X, Fang C, et al. Multiplex genome editing to generate universal CAR T cells resistant to PD1 inhibition. Clinical Cancer Res, 2016

26 Keener AB. Delivering the goods: scientists seek a way to make CRISPR-Cas gene editing more targeted. Nat Med, 2015, 21: 1239-1241

27 Tauxe W. Genome editing: 4 big questions. Nature, 2015, 528: S17-S17

28 Ran FA, Cong L, Yan WX, et al. In vivo genome editing using Staphylococcus aureus Cas9. Nature, 2015, 520: 186-191

29 Yin H, Kanasty RL, Eltoukhy AA, et al. Non-viral vectors for genebased therapy. Nat Rev Genet, 2014, 15: 541-555

30 Schmidt F, Grimm D. CRISPR genome engineering and viral gene delivery: a case of mutual attraction. Biotech J, 2015, 10: 258-272

31 Li L, Song L, Liu X, et al. Artificial virus delivers CRISPR-Cas9 system for genome editing of cells in mice. ACS Nano, 2016, doi: 10.1021/acsnano.6b04261

32 Zuris JA, Thompson DB, Shu Y, et al. Cationic lipid-mediated delivery of proteins enables efficient protein-based genome editing in vitro and in vivo. Nat Biotechnol, 2014, 33: 73-80

33 Dickel DE, Zhu Y, Nord AS, et al. Function-based identification of mammalian enhancers using site-specific integration. Nat Meth, 2014, 11: 566-571

34 Kim S, Kim D, Cho SW, et al. Highly efficient RNA-guided genome editing in human cells via delivery of purified Cas9 ribonucleoproteins. Genome Res, 2014, 24: 1012-1019

35 Ramakrishna S, Kwaku Dad AB, Beloor J, et al. Gene disruption by cell-penetrating peptide-mediated delivery of Cas9 protein and guide RNA. Genome Res, 2014, 24: 1020-1027

36 Wang M, Zuris JA, Meng F, et al. Efficient delivery of genomeediting proteins using bioreducible lipid nanoparticles. Proc Natl Acad Sci USA, 2016, 113: 2868-2873

37 Sun W, Ji W, Hall JM, et al. Self-assembled DNA nanoclews for the efficient delivery of CRISPR-Cas9 for genome editing. Angew Chem Int Ed, 2015, 54: 12029-12033

38 Sun W, Lu Y, Gu Z. Rolling circle replication for engineering drug delivery carriers. Therapeutic Deliver, 2015, 6: 765-768

39 Fu Y, Foden JA, Khayter C, et al. High-frequency off-target mutagenesis induced by CRISPR-Cas nucleases in human cells. Nat Biotechnol, 2013, 31: 822-826

40 Yin H, Song CQ, Dorkin JR, et al. Therapeutic genome editing by combined viral and non-viral delivery of CRISPR system components in vivo. Nat Biotechnol, 2016, 34: 328-333

41 Lu Y, Aimetti AA, Langer R, et al. Bioresponsive materials. Nat Rev Mater, 2016, 1: 16075

42 Sun W, Hu Q, Ji W, et al. Leveraging physiology for precision drug delivery. Physiol Rev, 2017, 97: 189-225

Acknowledgments This work was supported by the grants from North Carolina TraCS, NIH's Clinical and Translational Science Awards (CTSA, 1UL1TR001111) at UNC-CH, and Sloan Fellowship Award from the Alfred P. Sloan Foundation.

Author contributions Sun $\mathrm{W}$ and $\mathrm{Gu} \mathrm{Z}$ prepared the manuscript.

Conflict of interest The authors declare that they have no conflict of interest. 


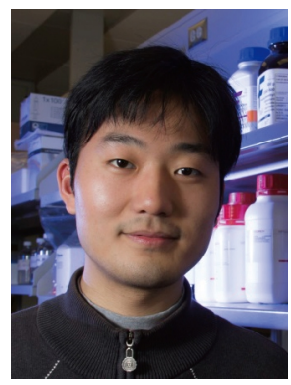

Wujin Sun received his BSs degree in bioengineering in 2010, followed by a MSc degree in biochemical engineering from Nanjing Tech University. He is currently a PhD student in Prof. Zhen Gu's laboratory in the Joint Department of Biomedical Engineering at the University of North Carolina (UNC) at Chapel Hill and North Carolina University. His current research is focused on the development of non-viral delivery carriers for the CRISPR-Cas9 system.

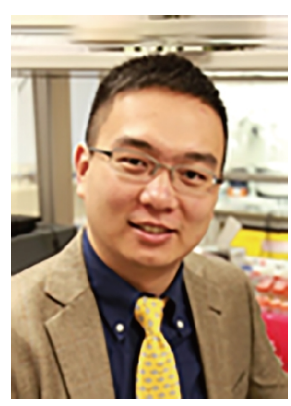

Zhen Gu obtained his PhD degree at the University of California, Los Angeles (UCLA), under the guidance of Prof. Yi Tang at the Department of Chemical and Biomolecular Engineering. He was a postdoctoral associate working with Prof. Robert Langer at MIT and Harvard Medical School. He is currently an associate professor at the Joint Department of Biomedical Engineering at the UNC at Chapel Hill and North Carolina State University. He also holds a joint position in the UNC Eshelman School of Pharmacy and UNC Department of Medicine. His group studies controlled drug delivery, bioinspired materials and nanobiotechnology.

\section{基因编辑系统非病毒载体的研究进展}

孙梧进 ${ }^{1,2}$, 顾瑧 $1,2,3^{*}$

摘要 II型CRISPR-Cas9系统业已成为一个风靡生物技术领域的基因编辑系统. 但将CRISPR-Cas9编辑系统直接用于活体来进行疾病治疗仍 受到来自递送载体的制约. 大分子量多组份的递运目标物, 加上向细胞核递送药物过程的多重生理屏障使得在活体中应用CRISPR-Cas9面 临挑战. 目前主流的递送体系是病毒载体, 但是出于对病毒载体安全性的考虑, 非病毒类载体亟待开发. 在这篇综述中, 我们总结了最近在 CRISPR-Cas9非病毒载体研究领域的进展并对其技术难点及未来发展方向展开了讨论. 